\title{
ADDENDUM, THE $q$-REGULARITY OF LATTICE POINT PATHS IN $R^{n}$
}

BY JEROME L. PAUL

Communicated July 19, 1975

Theorem 2 of [1] admits the following generalization.

THEOREM $2^{\prime} . \rho\left(Z^{n}\right) \leqslant n-1, n \geqslant 4$.

This result settles negatively a conjecture made in [1]. The partition of $Z^{n}$ verifying Theorem $2^{\prime}$ is given by $f \oplus g_{1} \oplus g_{2}$ when $n=4$, and $f \oplus g_{1} \oplus g_{2}$ $\oplus g_{4} \oplus \cdots \oplus g_{n-1}$ when $n \geqslant 5$, where the $g_{i}$ are defined by I, II, and III of [1], and $f: Z^{2} \rightarrow Z_{2}$ is defined by $f(x, y)=g_{3}(x)$ for $y \equiv 0(\bmod 2), f(x, y)=$ $g_{3} \tau_{3}(x)+1$ for $y \equiv 1(\bmod 4)$, and $f(x, y)=g_{3} \tau_{3}(x)$ for $y \equiv 3(\bmod 4)$. While $\rho\left(Z^{4}\right)=3$, the exact value of $\rho\left(Z^{n}\right)$ for $n \geqslant 5$ remains open.

\section{REFERENCE}

1. J. L. Paul, The q-regularity of lattice point paths in $R^{n}$, Bull. Amer. Math. Soc. 81 (1975), 492-494.

DEPARTMENT OF MATHEMATICAL SCIENCES, UNIVERSITY OF CINCINNATI, CINCINNATI, OHIO 45221 